\title{
Is Promoting Gut Microbial Diversity in Neonatal Enterocolitis the NECst Step?
}

\author{
Erika C. Claud ${ }^{1} \cdot$ Elaine O. Petrof $^{2}$
}

Published online: 12 October 2015

(C) Springer Science+Business Media New York 2015

\section{Introduction: The Gut Microbiome}

The human microbiome has substantial effects on health and disease. A diverse healthy microbiome has a resilient community composition that supports the host. For the preterm infant, microbiome development, contemporaneous with intestinal development, is influenced by clinical neonatal intensive care interventions [1,2].

\section{Necrotizing Enterocolitis (NEC)}

A prime example of neonatal disease associated with an altered gut microbiota diversity and composition, termed dysbiosis, is NEC. A devastating inflammatory bowel disease, NEC is the most common gastrointestinal (GI) emergency in the neonatal intensive care unit (ICU), with a mortality rate of $50 \%$ and no known cure [2]. NEC is the primary neonatal intestinal developmental disease, affecting $10 \%$ of infants with a birthweight $<1500 \mathrm{~g}$ [2]. The pathogenesis of NEC may be linked to alterations of the preterm infant microbiome. Although the primary NEC risk factors are prematurity and bacterial colonization, the influence of bacteria in the disease is unclear. To date, no single organism has been proven etiologic, despite extensive investigation [2]. Recently, microbial community

Elaine O. Petrof

eop@queensu.ca

1 Departments of Pediatrics and Medicine, University of Chicago, Chicago, IL 60637, USA

2 Gastrointestinal Diseases Research Unit, Department of Medicine, Queen's University, Kingston, ON K7L 2V7, Canada signatures associated with NEC were identified using advanced sequencing methods. In this issue of Digestive Diseases and Sciences, Leach et al. [3] measured the microbial composition and diversity of NEC babies over time compared with matched controls without NEC, also determining whether S100 calcium binding protein A12 (S100A12) can serve as a NEC biomarker. The authors reported that four organisms (Morganella morganii, Pseudomonas aeruginosa, Corynebacterium striatum, and Corynebacterium amycolatum) were more common in the NEC patients compared to controls, concluding that these opportunistic pathogens collectively contribute to the disease etiology.

\section{Comment}

Morganella morganii and Pseudomonas aeruginosa are well-described opportunistic pathogens, with the latter organism becoming more virulent when exposed to epinephrine and other environmental factors associated with host stress [4]. C. striatum and C. amycolatum are part of the normal human skin flora, and although increasingly recognized as potential pathogens in hospitalized and immunocompromised patient populations such as those suffering from endocarditis, bloodstream and catheter infections, empyema, and pneumonia [5], there are no reports in the literature of GI infections caused by these organisms. Although little is known about these organisms in the gut, a Danish study of upper jejunal fluid from 51 children with no known GI disease reported that $\mathrm{Co}$ rynebacterium $\mathrm{sp}$. were part of the normal gut flora in $10 \%$ of children aged 1-16 years, second only to Lactobacillus sp. in terms of abundance, although the organisms were not speciated further [6]. Therefore, the significance of these 
skin commensals toward NEC pathogenesis is unclear. Since all NEC babies received antibiotics, the presence of these organisms may be more due to the selective survival of Corynebacterium species under antimicrobial environmental pressures.

With only four NEC cases in the study, it is difficult to draw firm conclusions from such small numbers. The cases included were also quite heterogeneous: not only did birth weight range from 590 to $1410 \mathrm{~g}$ and gestational age range from 24 to 30 weeks, but furthermore two underwent surgical resection for acute NEC. The type and duration of antibiotics were not similar among the groups (for example, the duration of vancomycin/gentamicin administration ranged from 3 to 103 days), and mode of delivery and method of feeding were also variable, all of which likely alter the gut microbiome, introducing confounders that obscure broader interpretation of the data.

Nevertheless, their conclusions are consistent with other reports indicating that no one single organism causes NEC; it may be a mixture or combination of organisms that serves as the trigger. Other studies have shown changes in the relative composition of gut microbes (e.g., decreased Firmicutes and increased Proteobacteria, Enterobacter in babies with NEC), as well as overall decreased microbial diversity preceding the onset of NEC [7]. The combination of the four organisms described in this paper was not identified as prominent in other studies, highlighting the need for studies with larger sample sizes from multiple institutions that focus on microbial community function rather than solely on taxonomy.

S100A12, another focus of the study, is a fecal marker of intestinal inflammation that is a potential diagnostic biomarker for NEC [8]. Since there is currently no diagnostic test available for NEC, the authors revisited the question of whether SA can serve as a biomarker of NEC. Again, small numbers obscure any firm conclusions since serial stool samples were only available for $2 / 4$ of the NEC infants, with no indication of whether these were the two NEC infants that also underwent intestinal surgical resection.

\section{Future Directions}

Despite the limitations of the current study, it adds to the growing body of evidence that an aberrant microbiota is associated with NEC, with no single pathogen responsible for disease pathogenesis. Since intestinal dysbiosis and decreased microbial diversity both correlate with NEC, it stands to reason that optimization of the gut microbiota may be a logical next step in the future of NEC management and prevention. One option is manipulation of the microbiota, an approach well illustrated by the successes reported for probiotics. In a meta-analysis that examined over 5000 neonates, probiotics reduced severe NEC and all-cause mortality [9]. Approaches using complex microbial communities to restore damaged microbial ecosystems (effective at curing other diseases characterized by dysbiosis, such as recurrent $C$. difficile infection) are also intriguing, but have not been investigated in the preterm infant population [10]. Another more easily obtainable option may be protection of the developing microbiome through encouragement of exclusive human milk feeding and limitation of antibiotic use. Studies reporting dosedependent effects of decreased NEC with increased percent of human milk feeding and increased NEC with each additional day of early empiric antibiotic suggest the potential for protection with these interventions is comparable to effects seen with probiotic administration [11, 12]. More research focused on function of developing microbial communities is needed to understand how to define, optimize, and protect a normal healthy microbiota, in order to improve outcomes for preterm infants.

\section{References}

1. Koenig JE, Spor A, Scalfone N, Fricker AD, et al. Succession of microbial consortia in the developing infant gut microbiome. Proc Natl Acad Sci. 2011;108:4578-4585.

2. Neu J, Walker WA. Necrotizing enterocolitis. $N$ Engl J Med. 2011;364:255-264.

3. Leach ST, Lui K, Naing Z, Dowd SE, Mitchell HM, Day AS. Multiple opportunistic pathogens, but not pre-existing inflammation, may be associated with necrotizing enterocolitis. Dig Dis Sci. (Epub ahead of print). doi:10.1007/s10620-015-3830-6.

4. Alverdy J, Holbrook C, Rocha F, et al. Gut-derived sepsis occurs when the right pathogen with the right virulence genes meets the right host: evidence for in vivo virulence expression in Pseudomonas aeruginosa. Ann Surg. 2000;232:480-489.

5 . Bernard K. The genus corynebacterium and other medically relevant coryneform-like bacteria. J Clin Microbiol. 2012;50: $3152-3158$.

6. Justesen T, Nielsen OH, Hjelt K, Krasilnikoff PA. Normal cultivable microflora in upper jejunal fluid in children without gastrointestinal disorders. $J$ Pediatr Gastroenterol Nutr. 1984:3:683-686.

7. Wang Y, Hoenig JD, Malin KJ, et al. 16S rRNA gene-based analysis of fecal microbiota from preterm infants with and without necrotizing enterocolitis. ISME J. 2009;3:944-954.

8. Däbritz J, Jenke A, Wirth S, Foell D. Fecal phagocyte-specific S100A12 for diagnosing necrotizing enterocolitis. J Pediatr. 2012;161:1059-1064.

9. AlFaleh K, Anabrees J. Probiotics for prevention of necrotizing enterocolitis in preterm infants. Cochrane Database Syst Rev. 2014;4:CD005496.

10. Petrof EO, Claud EC, Gloor GB, Allen-Vercoe E. Microbial ecosystems therapeutics: a new paradigm in medicine? Benef Microbes. 2013;4:53-65.

11. Meinzen-Derr J, Poindexter B, Wrage L, Morrow AL, Stoll B, Donovan EF. Role of human milk in extremely low birth weight 
infants' risk of necrotizing enterocolitis or death. $J$ Perinatol. 2009;29:57-62.

12. Cotten CM, Taylor S, Stoll B, et al. Prolonged duration of initial empirical antibiotic treatment is associated with increased rates of necrotizing enterocolitis and death for extremely low birth weight infants. Pediatrics. 2009;123:58-66. 